\title{
Initial Boundary Value Problems for the Equations of Motion of Compressible Viscous and Heat-Conductive Fluids
}

\author{
Akitaka Matsumura ${ }^{1 \star}$ and Takaaki Nishida ${ }^{2 \star}$ \\ 1 Department of Applied Mathematics and Physics, Kyoto University, Kyoto 606, Japan \\ 2 Department of Mathematics, Kyoto University, Kyoto 606, Japan
}

\begin{abstract}
The equations of motion of compressible viscous and heat-conductive fluids are investigated for initial boundary value problems on the half space and on the exterior domain of any bounded region. The global solution in time is proved to exist uniquely and approach the stationary state as $t \rightarrow \infty$, provided the prescribed initial data and the external force are sufficiently small.
\end{abstract}

\section{Introduction}

The motion of viscous compressible fluids is described by the system of five equations for the density $\rho$, the velocity $u=\left(u^{1}, u^{2}, u^{3}\right)$ and the temperature $\theta$ :

$$
\begin{gathered}
\rho_{t}+\left(\rho u^{j}\right)_{x_{\jmath}}=0, \\
u_{t}^{i}+u^{j} u_{x_{\jmath}}^{i}+\frac{1}{\rho} p_{x_{\imath}}=\frac{1}{\rho}\left\{\mu\left(u_{x_{\jmath}}^{i}+u_{x_{t}}^{j}\right)+\mu^{\prime} u_{x_{k}}^{k} \delta^{i j}\right\}_{x_{\jmath}}+f^{i}, \quad i=1,2,3, \\
\theta_{t}+u^{j} \theta_{x_{\jmath}}+\frac{\theta p_{\theta}}{\rho c_{\nabla}} u_{\lambda_{\jmath}}^{j}=\frac{1}{\rho c_{\mathrm{V}}}\left\{\left(\kappa \theta_{x_{j}}\right)_{x_{J}}+\Psi\right\},
\end{gathered}
$$

where $p$ is the pressure, $\mu$ is the viscosity coefficient, $\mu^{\prime}$ is the second coefficient of viscosity, $\kappa$ is the coefficient of heat conduction, $c_{\nabla}$ is the specific heat at constant volume, all of which are known functions of $\rho$ and $\theta$, and $\Psi$ is the dissipation function:

$$
\Psi=\frac{\mu}{2}\left(u_{x_{k}}^{j}+u_{x_{j}}^{k}\right)^{2}+\mu^{\prime}\left(u_{x_{k}}^{k}\right)^{2} .
$$

We consider the initial boundary value problem of (1.1) in the region $t \geqq 0, x \in \Omega$. The boundary condition is supposed

$$
\left.u\right|_{\partial \Omega}=\left.u\right|_{\infty}=0,\left.\quad \theta\right|_{\partial \Omega}=\left.\theta\right|_{\infty}=\bar{\theta}, \quad t>0,
$$

* Both authors are partially supported by the University of Wisconsin-Madison, Mathematics Research Center 
or

$$
\left.u\right|_{\partial \Omega}=\left.u\right|_{\infty}=0,\left.\quad \frac{\partial \theta}{\partial n}\right|_{\partial \Omega}=\left.\frac{\partial \theta}{\partial n}\right|_{\infty}=0, \quad t>0 .
$$

The initial condition is given by

$$
(\rho, u, \theta)(0, x)=\left(\rho_{0}, u_{0}, \theta_{0}\right)(x), \quad x \in \Omega .
$$

The local existence theorem for the initial boundary value problem (1.1)-(1.4) is proved by Tani [10] under full generality. Here we want to solve the problem (1.1)(1.4) globally in time under the following assumptions.

A.1. The domain $\Omega$ is the half space $R_{+}^{3}=\left\{x \in R^{3}, x_{3}>0\right\}$ or an exterior domain of any bounded region with smooth boundary. Here we note that the initial (-boundary) value problems on the whole space and on the interior domain are solved globally in time in [6-8].

A.2. $\mu, \mu^{\prime}, \kappa, p$ and $c_{\nabla}$ are smooth functions of $\rho, \theta>0$, and $\mu, \kappa, c_{\nabla}, p, p_{\rho}, p_{\theta}>0$, $\mu^{\prime}+(2 / 3) \mu \geqq 0$.

A.3. The external force $f$ is given by the potential $\Phi(x) \in H^{5}(\Omega)$,

$$
f^{\prime}=-\Phi_{x_{i}}, \quad i=1,2,3, \quad x \in \Omega
$$

where, and in what follows, we use the Sobolev space

$$
H^{l}(\Omega)=\left\{f \in L_{2}(\Omega), D^{k} f=\left\{\partial^{\alpha} f / \partial x_{1}^{\alpha_{1}} \partial x_{2}^{\alpha_{2}} \partial x_{3}^{\alpha_{3}},|\alpha|=k\right\} \in L_{2}(\Omega), 1 \leqq k \leqq l\right\}
$$

with the norm $\|f\|_{l}=\left(\sum_{k=0}^{l} \int_{\Omega}\left|D^{k} f\right|^{2} d x\right)^{1 / 2}$.

A.4. The initial data are smooth functions close to a constant state $(\bar{\rho}, 0, \bar{\theta})$, where $\bar{\rho}$ and $\bar{\theta}$ (also appeared in (1.2)) are any positive constants, i.e.,

$$
\rho_{0}-\bar{\rho}, u_{0}, \theta_{0}-\bar{\theta} \in H^{3}(\Omega) \text {, and }\left\|\rho_{0}-\bar{\rho}, u_{0}, \theta_{0}-\bar{\theta}\right\|_{3} \text { is small. }
$$

A.5. The compatibility condition on the initial and boundary data is satisfied as follows:

$$
\begin{aligned}
& \left.u_{0}\right|_{\partial \Omega}=0,\left.\quad \theta_{0}\right|_{\partial \Omega}=\bar{\theta}, \\
& -\frac{p_{0, x_{2}}}{\rho_{0}}+\left.\frac{1}{\rho_{0}}\left\{\mu_{0}\left(u_{0, x_{\jmath}}^{i}+u_{0, x_{\iota}}^{j}\right)+\mu_{0}^{\prime} u_{0, x_{h}}^{k} \delta^{i j}\right\}_{x \jmath}\right|_{\partial \Omega}=\left.\Phi_{x_{\imath}}\right|_{\partial \Omega}, \\
& -\frac{\theta_{0}\left(p_{\theta}\right)_{0}}{\rho_{0}\left(c_{\nabla}\right)_{0}} u_{0, x_{j}}^{j}+\left.\frac{1}{\rho_{0}\left(c_{\nabla}\right)_{0}}\left\{\left(\kappa_{0} \theta_{0, k_{\jmath}}\right)_{x_{\jmath}}+\Psi_{0}\right\}\right|_{\partial \Omega}=0,
\end{aligned}
$$

where we used the notation $p_{0}=p\left(\rho_{0}, \theta_{0}\right)$ etc. In the case of Neumann condition (1.3) for $\theta$ we need a necessary change of compatibility condition.

We use the following notations for the function spaces:

$\mathscr{C}^{0}(\bar{\Omega})$ : Banach space of bounded continuous function on $\bar{\Omega}$,

$\mathscr{C}^{l}(\bar{\Omega})=\left\{f \in \mathscr{C}^{0}(\bar{\Omega}), D^{k} f \in \mathscr{C}^{0}(\bar{\Omega}), 1 \leqq k \leqq l\right\}$,

$\mathscr{C}^{l}\left(t_{1}, t_{2} ; B\right)=\{u(t): \quad$-time continuously differentiable function of 
$t \in\left[t_{1}, t_{2}\right]$ with values in a Banach space $B$, where the norm is given by $\left.\max \sup \left\|(\partial / \partial t)^{k} u(t, \cdot)\right\|_{B}\right\}$,

$0 \leqq k \leqq l t_{1} \leqq t \leqq t_{2}$

$L_{2}\left(t_{1}, t_{2} ; B\right)=\left\{u(t): L_{2}\right.$-function of $t \in\left[t_{1}, t_{2}\right]$ with values in $B$, where the norm is given by $\left.\left\{\int_{t_{1}}^{t_{2}}\|u(t, \cdot)\|_{B}^{2} d t\right\}^{1 / 2}\right\}$.

The stationary solution $(\tilde{\rho}, \tilde{u}, \tilde{\theta})(x)$ of $(1.1)-(1.3)$ in a neighborhood of $(\bar{\rho}, 0, \bar{\theta})$ in $H^{2}(\Omega) \times H^{2}(\Omega) \times H^{2}(\Omega)$ has the form (cf. Lemma 2.1)

$$
\int_{\bar{\rho}}^{\tilde{\rho}(x)} \frac{p_{\rho}(\eta, \bar{\theta})}{\eta} d \eta+\Phi(x)=0, \quad \tilde{u}=0, \tilde{\theta}=\bar{\theta} .
$$

We can state the main

Theorem 1.1. Under the Assumptions A.1. $\sim$ A.5 there exists a constant $\varepsilon>0$ such that if

$$
\left\|\rho_{0}-\bar{\rho}, u_{0}, \theta_{0}-\bar{\theta}\right\|_{3}+\|\Phi\|_{5} \leqq \varepsilon
$$

then the initial boundary value problem (1.1)-(1.4) has a unique solution $(\rho, u, \theta)$ globally in time and a unique stationary state $(\tilde{\rho}, 0, \bar{\theta})$, which satisfy

$$
\begin{array}{r}
\rho-\tilde{\rho} \in \mathscr{C}^{0}\left(0, \infty ; H^{3}(\Omega)\right) \cap \mathscr{C}^{1}\left(0, \infty ; H^{2}(\Omega)\right), \\
u, \theta-\bar{\theta} \in \mathscr{C}^{0}\left(0, \infty ; H^{3}(\Omega)\right) \cap \mathscr{C}^{1}\left(0, \infty ; H^{1}(\Omega)\right), \\
|\rho-\tilde{\rho}, u, \theta-\bar{\theta}(t)|_{\mathscr{C}^{0}(\bar{\Omega})} \rightarrow 0 \text { as } t \rightarrow \infty .
\end{array}
$$

The theorem is proved by the energy method which is similar to [6-8], but it requires the estimates valid in the half space and in the exterior domain. We note that the solution decays as in (1.10), but the decay rate is not known, cf. [6-8]. In the following we only mention the arguments for the Dirichlet boundary condition (1.2), because the Neumann boundary condition (1.3) for $\theta$ can be treated similarly.

\section{Stationary Solution}

Let us write the equations and conditions for the stationary solution $(\tilde{\rho}, \tilde{u}, \tilde{\theta})$ :

$$
\begin{gathered}
\left(\tilde{\rho} \tilde{u}^{j}\right)_{x_{\jmath}}=0, \\
\tilde{\rho} \tilde{u}^{j} \tilde{u}_{x_{\jmath}}^{i}+\tilde{p}_{x_{l}}+\tilde{\rho} \Phi_{x_{\imath}}-\left(\tilde{\mu}\left(\tilde{u}_{x_{1}}^{i}+\tilde{u}_{x_{l}}^{j}\right)+\tilde{\mu}^{\prime} \tilde{u}_{x_{k}}^{k} \delta^{i j}\right)_{x_{\jmath}}=0, i=1,2,3, \\
\tilde{\rho} \tilde{c}_{\nabla} \tilde{u}^{j} \tilde{\theta}_{x_{\jmath}}+\tilde{\theta} \tilde{p}_{\theta} u_{x_{\jmath}}^{j}-\left(\tilde{\kappa} \tilde{\theta}_{x_{\jmath}}\right)_{x_{\jmath}}-\tilde{\Psi}=0, \\
\left.\tilde{u}\right|_{\hat{c} \Omega}=\left.\tilde{u}\right|_{\infty}=0,\left.\quad \tilde{\theta}\right|_{\partial \Omega}=\left.\tilde{\theta}\right|_{\infty}=\bar{\theta},\left.\quad \tilde{\rho}\right|_{\infty}=\bar{\rho},
\end{gathered}
$$

where $\tilde{p}=p(\tilde{\rho}, \tilde{\theta})$ etc. The stationary problem (2.1)-(2.4) has a unique solution as

Lemma 2.1. Under the assumptions A.1 $\sim$ A.3 there exist positive constants $\varepsilon$ and $C$ such that if $\|\Phi\|_{l} \leqq \varepsilon, l=3,4$ or 5 the problem (2.l)-(2.4) has a unique solution $(\tilde{\rho}(x), 0$, $\bar{\theta})$ in a small neighborhood of $(\tilde{\rho}, 0, \tilde{\theta})$ in $\mathrm{H}^{2} \times \mathrm{H}^{2} \times \mathrm{H}^{2}$ satisfying

$$
\|\tilde{\rho}-\bar{\rho}\|_{l} \leqq C\|\Phi\|_{l}, l=3,4 \text { or } 5 \text { respectively, }
$$


where $\tilde{\rho}(x)$ is determined by (1.8), i.e.,

$$
\int_{\bar{\rho}}^{\tilde{\rho}(x)} \frac{p_{\rho}(\eta, \bar{\theta})}{\eta} d \eta+\Phi(x)=0 .
$$

Proof. Since we consider a small neighborhood of $(\bar{\rho}, 0, \bar{\theta})$ in $H^{2} \times H^{2} \times H^{2}$, by Sobolev's lemma, we may suppose $|\tilde{\rho}-\bar{\rho}|,|\tilde{u}|,|\tilde{\theta}-\bar{\theta}|<\frac{1}{2} \min \{\bar{\rho}, \bar{\theta}\}$. Then we can estimate the equalities:

$$
\begin{aligned}
& \int[2.1] \times \int_{\bar{\rho}}^{\tilde{\rho}} \frac{p_{\rho}(\eta, \bar{\theta})}{\eta} d \eta d x=0, \\
& \int[2.2]^{i} \times \tilde{u}^{i} d x=0, \\
& \int[2.3] \times[\bar{\theta}-\bar{\theta}) d x=0,
\end{aligned}
$$

where [2.1], [2.2 $]^{i}$ and [2.3] denote the terms on the left hand side of $(2.1),(2.2)^{i}$ and (2.3) respectively. Take the sum of (2.7) and integrate it by parts using the mean value theorem and Lemma 4.1. We obtain the inequality:

$$
\|D \tilde{u}\|^{2}+\|D \tilde{\theta}\|^{2} \leqq C\left\{\|D \tilde{\rho}\|+\|\tilde{u}\|_{1}+|\tilde{\theta}-\bar{\theta}|_{\mathscr{C}_{0}}+\|\tilde{\theta}-\bar{\theta}\|\right\}\left(\|D \tilde{u}\|^{2}+\|D \tilde{\theta}\|^{2}\right) .
$$

Therefore if $\|D \tilde{\rho}\|,\|\tilde{u}\|_{1},\|\tilde{\theta}-\bar{\theta}\|_{2}$ is small, we can conclude

$$
\tilde{u}=0, \tilde{\theta}=\bar{\theta} .
$$

If we substitute $(2.8)$ in (2.2), we have

$$
\left\{\int_{\bar{\rho}}^{\tilde{\rho}} \frac{p_{\rho}(\eta, \bar{\theta})}{\eta} d \eta+\Phi\right\}_{x_{\imath}}=0,
$$

which implies (2.6).

\section{Local and Global Existence}

Let us rewrite the problem (1.1), (1.2) by the change of variables $(\rho, u, \theta) \rightarrow$ $(\rho+\tilde{\rho}, u, \theta+\bar{\theta})$ using $(2.6)$ as follows:

$$
\begin{gathered}
\rho_{t}+u^{j} \rho_{x_{J}}+(\rho+\tilde{\rho}) u_{x_{j}}^{j}+\tilde{\rho}_{x_{J}} u^{j}=0, \\
u_{t}^{i}+u^{j} u_{x_{\jmath}}^{i}-\frac{1}{\rho+\tilde{\rho}}\left\{\mu\left(u_{x_{J}}^{i}+u_{x_{i}}^{j}\right)+\mu^{\prime} u_{x_{k}}^{k} \delta^{i j}\right\}_{x_{j}} \\
+\frac{p_{\rho}}{\rho+\tilde{\rho}} \rho_{x_{i}}+\frac{p_{\theta}}{\rho+\tilde{\rho}} \theta_{x_{i}}=\left(\frac{\tilde{\rho} p_{\rho}(\rho+\tilde{\rho}, \theta+\bar{\theta})}{(\rho+\tilde{\rho}) p_{\rho}(\tilde{\rho}, \bar{\theta})}-1\right) \Phi_{x_{i}}, \\
\theta_{t}+u^{j} \theta_{x_{J}}+\frac{(\theta+\bar{\theta}) p_{\theta}}{(\rho+\bar{\rho}) c_{\nabla}} u_{x_{j}}^{j}=\frac{1}{(\rho+\bar{\rho}) c_{\nabla}}\left\{\left(\kappa \theta_{x_{j}}\right)_{x_{j}}+\Psi\right\}, \\
\left.(u, \theta)\right|_{\partial \Omega}=\left.(u, \theta)\right|_{\infty}=0, \\
(\rho, u, \theta)(0, x)=\left(\rho_{0}, u_{0}, \theta_{0}\right)(x) .
\end{gathered}
$$


Further we rewrite the problem (3.1)-(3.3) as follows:

$$
\begin{gathered}
L_{u}^{0}(\rho, u) \equiv \rho_{t}+u^{j} \rho_{x_{j}}+\bar{\rho} u_{x_{j}}^{j}=f^{0}, \\
L^{i}(\rho, u, \theta) \equiv u_{t}^{i}-\hat{\mu} u_{x_{j} x_{j}}^{i}-\left(\hat{\mu}+\hat{\mu}^{\prime}\right) u_{x_{i} x_{j}}^{j}+p_{1} \rho_{x_{\imath}}+p_{2} \theta_{x_{\imath}}=f^{i}, i=1,2,3, \\
L^{4}(u, \theta) \equiv \theta_{t}-\hat{\kappa} \theta_{x_{j} x_{j}}+p_{3} u_{x_{\jmath}}^{j}=f^{4}, \\
\left.(u, \theta)\right|_{\partial \Omega}=\left.(u, \theta)\right|_{\infty}=0, \\
(\rho, u, \theta)(0)=\left(\rho_{0}, u_{0}, \theta_{0}\right),
\end{gathered}
$$

where we denote the constant for the function $g$ of $\rho$ and $\theta$ by $\bar{g}=g(\bar{\rho}, \bar{\theta})$, and also $\hat{\mu}=\bar{\mu} / \bar{\rho}, \hat{\mu}^{\prime}=\bar{\mu}^{\prime} / \bar{\rho}, \hat{\kappa}=\bar{\kappa} / \bar{\rho} \bar{c}_{\nabla}, p_{1}=\bar{p}_{\rho} / \bar{\rho}, p_{2}=\bar{p}_{\theta} / \bar{\rho}$ and $p_{3}=\bar{\theta} \bar{p}_{\theta} / \bar{\rho} \bar{c}_{\nabla}$. The terms on the right hand side of Eqs. (3.4) are nonlinear and have the form:

$$
\begin{aligned}
& f^{0}(\rho, u, \theta) \equiv(\bar{\rho}-\rho-\tilde{\rho}) u_{x_{J}}^{j}-\tilde{\rho}_{x_{j}} u^{j}, \\
& f^{i}(\rho, u, \theta) \equiv-u^{j} u_{x_{\jmath}}^{i}+\left(\frac{\mu}{\rho+\tilde{\rho}}-\hat{\mu}\right) u_{x_{\jmath} x_{J}}^{i} \\
& +\left(\frac{\mu+\mu^{\prime}}{\rho+\tilde{\rho}}-\hat{\mu}-\hat{\mu}^{\prime}\right) u_{x_{i} x_{\jmath}}^{j}+\frac{1}{\rho+\tilde{\rho}}\left\{\mu_{x_{\jmath}}\left(u_{x_{\jmath}}^{i}+u_{x_{i}}^{j}\right)\right. \\
& \left.+\mu_{x_{J}}^{\prime} u_{x_{k}}^{k} \delta^{i j}\right\}+\left(p_{1}-\frac{p_{\rho}}{\rho+\tilde{\rho}}\right) \rho_{x_{i}}+\left(p_{2}-\frac{p_{\theta}}{\rho+\tilde{\rho}}\right) \theta_{x_{i}} \\
& +\left(\frac{\tilde{\rho} p_{\rho}(\rho+\tilde{\rho}, \theta+\bar{\theta})}{(\rho+\tilde{\rho}) p_{\rho}(\tilde{\rho}, \bar{\theta})}-1\right) \Phi_{x_{1}}, \quad i=1,2,3, \\
& f^{4}(\rho, u, \theta) \equiv-u^{j} \theta_{x_{J}}+\left(\frac{\kappa}{(\rho+\tilde{\rho}) c_{\nabla}}-\hat{\kappa}\right) \theta_{x_{j} x_{j}} \\
& +\left(p_{3}-\frac{(\theta+\bar{\theta}) p_{\theta}}{(\rho+\tilde{\rho}) c_{\nabla}}\right) u_{x_{j}}^{j}+\frac{1}{(\rho+\tilde{\rho}) c_{\bar{\nabla}}}\left(\kappa_{x_{j}} \theta_{x_{j}}+\Psi\right) .
\end{aligned}
$$

Next we choose a constant $E_{0}$ by use of Sobolev's lemma such that

$$
\|g\|_{C^{0}} \leqq \frac{1}{2} \min (\bar{\rho}, \bar{\theta}) \text { for any } g \in H^{2},\|g\|_{2} \leqq E_{0} .
$$

Then the solution of (3.1)-(3.3) is sought in the set of functions $X(0, \infty ; E)$ for some $E \leqq E_{0}$, where for $0 \leqq t_{1} \leqq t_{2}<\infty$, we define

where

$$
\begin{aligned}
& X\left(t_{1}, t_{2} ; E\right)=\{(\rho, u, \theta): \\
& \rho \in \mathscr{C}\left(t_{1}, t_{2} ; H^{3}\right), D \rho \in L_{2}\left(t_{1}, t_{2} ; H^{2}\right), \\
& \rho_{t} \in \mathscr{C}\left(t_{1}, t_{2} ; H^{2}\right) \cap L_{2}\left(t_{1}, t_{2} ; H^{2}\right), \\
& \quad u, \theta \in \mathscr{C}\left(t_{1}, t_{2} ; H^{3} \cap H^{1}\right), D(u, \theta) \in L_{2}\left(t_{1}, t_{2} ; H^{3}\right), \\
& \left.u_{t}, \theta_{t} \in \mathscr{C}\left(t_{1}, t_{2} ; H^{1}\right) \cap L_{2}\left(t_{1}, t_{2} ; H^{2}\right) \text { and } N\left(t_{1}, t_{2}\right) \leqq E\right\},
\end{aligned}
$$

$$
\begin{aligned}
N^{2}\left(t_{1}, t_{2}\right) \equiv & \sup _{t_{\mathrm{r}} \leqq t \leqq t_{2}}\|\rho, u, \theta(t)\|_{3}^{2}+\left\|\rho_{t}(t)\right\|_{2}^{2}+\left\|u_{t}, \theta_{t}(t)\right\|_{1}^{2} \\
& +\int_{t_{1}}^{t_{2}}\|D \rho(s)\|_{2}^{2}+\left\|\rho_{t}(s)\right\|_{2}^{2}+\|D(u, \theta)(s)\|_{3}^{2}+\left\|u_{t}, \theta_{t}(s)\right\|_{2}^{2} d s .
\end{aligned}
$$


Here and in what follows we do not write $\Omega$ in $H^{l}(\Omega)$ and $L_{2}(\Omega)$.

We will obtain the global solution by a combination of a local existence theorem and some a priori estimates for the solution in $X$, namely that for the norm $N$.

Proposition 3.1. (Local Existence). Suppose the problem (3.1)-(3.3) has a unique solution $(\rho, u, \theta) \in X\left(0, h ; E_{0}\right)$ for some $h \geqq 0$, and consider the problem (3.I)-(3.3) for $t \geqq h$. Then there exist positive constants $\tau$, $\varepsilon_{0}$ and $C_{0}\left(\varepsilon_{0} \sqrt{1+C_{0}^{2}} \leqq E_{0}\right)$ independent of $h$ such that if $N(h, h),\|\Phi\|_{4} \leqq \varepsilon_{0}$, the problem has a unique solution $(\rho, u, \theta) \in$ $X\left(h, h+\tau ; C_{0} N(h, h)\right)$.

The proof is the same as that for the interior problem in [8] and is omitted. Although the local existence theorem by Tani [10] is more general, we need it in the form of Proposition 3.1 to extend the solution globally in time by use of the $L_{2}$ energy method.

Proposition 3.2. (a priori Estimates). Suppose the problem (3.1)-(3.3) has a solution $(\rho, u, \theta) \in X\left(0, h ; E_{0}\right)$ for given $h>0$. Then there exist positive constants $\varepsilon_{1}$ and $C_{1}\left(\varepsilon_{1} \leqq \varepsilon_{0}, \varepsilon_{1} C_{1} \leqq E_{0}\right)$ which are independent of $h$ such that if $N(0, h),\|\Phi\|_{5} \leqq \varepsilon_{1}$, then $N(0, h) \leqq C_{1} N(0,0)$.

If Proposition 3.1 and 3.2 are known, then global existence of a unique solution can be proven as follows: Choose the initial data $\left(\rho_{0}, u_{0} \theta_{0}\right)$ and the potential function $\Phi$ so small that

$$
N(0,0) \leqq \min \left\{\varepsilon_{0}, \varepsilon_{1} / C_{0}, \varepsilon_{1} / C_{1} \sqrt{1+C_{0}^{2}}\right\} \text { and }\|\Phi\|_{5} \leqq \varepsilon_{1} .
$$

The Proposition 3.1 with $h=0$ gives a local solution $(\rho, u, \theta) \in X\left(0, \tau ; C_{0} N(0,0)\right)$. Since $C_{0} N(0,0) \leqq \varepsilon_{1} \leqq \varepsilon_{0}$, Proposition 3.2 with $h=\tau$ implies $N(0, \tau) \leqq C_{1} N(0,0)$. Then Proposition 3.1 with $h=\tau$ implies the existence of a solution

$$
(\rho, u, \theta) \in X\left(\tau, 2 \tau ; C_{0} N(\tau, \tau)\right), \quad \in X\left(0,2 \tau ; \sqrt{1+C_{0}^{2}} N(0, \tau)\right) .
$$

Hence, since $\sqrt{1+C_{0}^{2}} N(0, \tau) \leqq C_{1} \sqrt{1+C_{0}^{2}} N(0,0) \leqq \varepsilon_{1}$, Proposition 3.2 with $h=2 \tau$ gives $N(0,2 \tau) \leqq C_{1} N(0,0)$, and Proposition 3.1 with $h=2 \tau$ gives

$$
(\rho, u, \theta) \in X\left(2 \tau, 3 \tau ; C_{0} N(2 \tau, 2 \tau)\right), \quad \in X\left(0,3 \tau ; \sqrt{1+C_{0}^{2}} N(0,2 \tau)\right)
$$

Repetition of this process yields

Proposition 3.3. (Global Existence). There exist positive constants $\varepsilon$ and $C\left(\varepsilon C \leqq E_{0}\right)$ such that if $N(0,0),\|\Phi\|_{5} \leqq \varepsilon$, then the initial boundary value problem (3.I)-(3.3) has a unique solution $(\rho, u, \theta) \in X(0, \infty ; C N(0,0))$.

\section{A Priori Estimates}

First we recall some inequalities of Sobolev type.

\section{Lemma 4.1.}

(i) Let $\Omega$ be any domain with smooth boundary. Then

$$
\begin{gathered}
\|f\|_{\mathscr{C}^{\sigma}(\Omega)} \leqq C\|f\|_{H^{2}(\Omega)}, \quad 0 \leqq \sigma<1 / 2 \\
\|f\|_{L_{p}(\Omega)} \leqq C\|f\|_{H^{1}(\Omega)}, \quad 2 \leqq p \leqq 6
\end{gathered}
$$


(ii) Let $\Omega$ be the whole space $R^{3}$, the half space $R_{+}^{3}$, or the exterior domain of a bounded region with smooth boundary. Then

$$
\|f\|_{L_{6}(\Omega)} \leqq C\|D f\|_{L_{2}(\Omega)}, \quad\|f\|_{L_{2}\left(\Omega^{\prime}\right)} \leqq C\|D f\|_{L_{2}(\Omega)},
$$

where $\Omega^{\prime}$ is any bounded subregion of $\Omega$.

Proof. See for example [3], [4].

Next we note some estimates of the elliptic system of equations for our domain, when we regard Eq. $(3.4)^{i}, i=1, \ldots, 4$, as elliptic with respect to $x$ variables, i.e.,

$$
\begin{aligned}
\hat{\mu} u_{x_{\jmath} x_{\jmath}}^{i}+\left(\hat{\mu}+\hat{\mu}^{\prime}\right) u_{x_{t} x_{\jmath}}^{j} & =u_{t}^{i}+p_{1} \rho_{x_{t}}+p_{2} \theta_{x_{t}}-f^{i}, \quad i=1,2,3, \\
\hat{\kappa} \theta_{x_{\jmath} x_{J}} & =\theta_{t}+p_{3} u_{x_{J}}^{j}-f^{4} \\
\left.u\right|_{\partial \Omega} & =\left.u\right|_{\infty}=\left.\theta\right|_{\partial \Omega}=\left.\theta\right|_{\infty}=0 .
\end{aligned}
$$

Lemma 4.2. Let $\Omega$ be the half space or any exterior domain. We have for $k=2,3$,

$$
\begin{aligned}
& \left\|D^{k} u\right\| \leqq C\left\{\left\|u_{t}\right\|_{k-2}+\|D(\rho, \theta)\|_{k-2}+\|f\|_{k-2}+\|u\|\right\}, \\
& \left\|D^{k} \theta\right\| \leqq C\left\{\left\|\theta_{1}\right\|_{k-2}+\|D u\|_{k-2}+\|f\|_{k-2}+\|D \theta\|\right\} .
\end{aligned}
$$

The first estimate is well known, e.g. [1]. The last $L_{2}$ norm is contained on the right hand side because of the unboundedness of our domain. The second one is given in [5]. It contains the $L_{2}$ norm of the first derivative as the last term on the right hand side and is stronger than that containing the $L_{2}$ norm of the function itself such as (4.4).

The last estimate for an elliptic system concerns stokes equation in $\Omega$ which comes from $(3.4)^{i}, i=0, \ldots, 4$.

$$
\begin{aligned}
\bar{\rho} u_{x_{\jmath}}^{j} & =h, \\
-\hat{\mu} u_{x_{\jmath} x_{\jmath}}^{i}+p_{1} \rho_{x_{i}} & =g^{i}, \quad i=1,2,3, \\
\left.u\right|_{\partial \Omega} & =a,\left.\quad u\right|_{\infty}=0 .
\end{aligned}
$$

Lemma 4.3. For $k=2,3,4$,

$$
\left\|D^{k} u\right\|^{2}+\left\|D^{k-1} \rho\right\|^{2} \leqq C\left\{\|h\|_{k-1}^{2}+\|g\|_{k-2}^{2}+\|a\|_{H^{k-1 / 2(\partial \Omega)}}^{2}+\|D u\|^{2}\right\},
$$

where the last term on the right hand side is necessary in the case of exterior domain.

Proof. In the case of the half space $\Omega=R_{3}^{+}$, the lemma is proved by Solonnikov [9] and Cattabriga [2], where the definition of the space $H^{k-1 / 2}(\partial \Omega)$ is also given. In the case of exterior domains Finn [3] and Heywood [4] obtain the lemma provided $h=0$. Thus we only need a slight improvement for $h \neq 0$. Let us extend the function $h \in H^{1}(\Omega)$ to a function $\tilde{h} \in H^{1}\left(R^{3}\right)$ with the estimate $\|\tilde{h}\|_{H^{1}\left(R^{3}\right)} \leqq C\|h\|_{H^{1}(\Omega)}$. Then we approximate $\tilde{h}$ by $H^{1}$ functions $h_{n}$ with compact support, i.e., $h_{n} \rightarrow \tilde{h}$ in $H^{1}\left(R^{3}\right)$, as $n \rightarrow \infty$. Thus we can define the function

$$
\phi_{n}(x)=\frac{1}{\bar{\rho}} \int_{R^{3}} \frac{h_{n}(y)}{|x-y|} d y .
$$


It is well known by use of Fourier transform

$$
\left\|D^{2} \phi_{n}\right\|_{H^{1}\left(R^{3}\right)} \leqq C\left\|h_{n}\right\|_{H^{1}\left(R^{3}\right)} .
$$

Thus we have

$$
\left\|D^{2} \phi_{n}\right\|_{H^{1}(\Omega)},\left\|D \phi_{n}\right\|_{L_{2}\left(\Omega^{\prime}\right)} \leqq C\left\|h_{n}\right\|_{H^{1}\left(R^{3}\right)}
$$

where $\Omega^{\prime}$ is any bounded subdomain of $\Omega$ (cf. (4.2)). Since $C$ is independent of $n$, as the limit of $n \rightarrow \infty$, we have

$$
\left\|D^{2} \phi\right\|_{H^{1}(\Omega)},\|D \phi\|_{L_{2}\left(\Omega^{\prime}\right)} \leqq C\|h\|_{H^{1}(\Omega)} .
$$

Now we can put $u-\nabla \phi=v$ and $q=\rho+h / p_{1} \bar{\rho}$, which satisfy $\operatorname{div} v=0$, $-\hat{\mu} \Delta v+p_{1} \nabla q=g$, and reduces to the above case. In fact we have

$$
\|D v\|_{L_{2}(\Omega)} \leqq\|D u\|_{L_{2}(\Omega)}+\left\|D^{2} \phi\right\|_{L_{2}(\Omega)} \leqq\|D u\|_{L_{2}(\Omega)}+C\|h\|_{H^{1}(\Omega)},
$$

and

$$
\left\|\left.v\right|_{\hat{\partial} \Omega}\right\|_{H^{3 / 2}(\partial \Omega)}=\left\|-\left.\nabla \phi\right|_{\partial \Omega}\right\|_{H^{3 / 2}(\partial \Omega)} \leqq C\|D \phi\|_{H^{2}\left(\Omega^{\prime}\right)} \leqq C\|h\|_{H^{1}(\Omega)},
$$

where the boundary of $\Omega^{\prime}$ contains $\partial \Omega$.

Now we begin to obtain the energy estimate for solution of Eq. $(3.4)^{i}, i=0, \ldots, 4$, with (3.5).

Lemma 4.4. We have for $l=0$ and 1

where

$$
\begin{aligned}
& \left\|\partial_{t}^{l}(\rho, u, \theta)(t)\right\|^{2}+\int_{0}^{t}\left\|D \partial_{t}^{l}(u, \theta)(s)\right\|^{2}+\left\|\partial_{t}^{l} \frac{d \rho}{d t}(s)\right\|^{2} d s \\
& \leqq C\left\{\left\|\partial_{t}^{l}(\rho, u, \theta)(0)\right\|^{2}+\int_{0}^{t}\left|A_{l}\right|+\left\|\partial_{t}^{l} f^{0}\right\|^{2} d s\right\},
\end{aligned}
$$

$$
\begin{aligned}
& A_{0}=\int \frac{p_{1}}{\bar{\rho}} \rho\left(f^{0}-u^{j} \rho_{x_{J}}\right)+u^{i} f^{i}+\frac{p_{2}}{p_{3}} \theta f^{4} d x, \\
& A_{1}=\int \frac{p_{1}}{\bar{\rho}} \rho_{t}\left(f^{0}-u^{j} \rho_{x_{j}}\right)_{t}+u_{t}^{i} f_{t}^{i}+\frac{p_{2}}{p_{3}} \theta_{t} f_{t}^{4} d x, \\
& \frac{d \rho}{d t} \equiv \rho_{t}+u^{j} \rho_{x_{J}}=f^{0}-\bar{\rho} u_{x_{j}}^{j} .
\end{aligned}
$$

We have also for $k=0$ and 1 ,

$$
\begin{aligned}
\left\|D \partial_{t}^{k}(u, \theta)(t)\right\|^{2} & +\int_{0}^{t}\left\|\partial_{t}^{k+1}(\rho, u, \theta)(s)\right\|^{2} d s \leqq C\left\{\left\|D \partial_{t}^{k}(u, \theta)(0)\right\|^{2}\right. \\
& +\left\|\partial_{t}^{k} \rho(0)\right\|^{2}+\left\|\partial_{t}^{k} \rho(t)\right\|^{2}+\int_{0}^{t}\left\|D \partial_{t}^{k}(u, \theta)(s)\right\|^{2} \\
& \left.+\left\|\partial_{t}^{k}\left(f^{0}-u^{j} \rho_{x_{j}}\right)\right\|^{2}+\left\|\partial_{t}^{k} f(s)\right\|^{2} d s\right\} .
\end{aligned}
$$


Proof. Compute the integral

$$
\int_{0}^{t} \int_{\Omega} \frac{p_{1}}{\bar{\rho}} \rho\left(L^{0}-f^{0}\right)+u^{i}\left(L^{i}-f^{i}\right)+\frac{p_{2}}{p_{3}} \theta\left(L^{4}-f^{4}\right) d x d t=0 .
$$

Integration by parts using the boundary condition gives

$$
\begin{aligned}
& \frac{1}{2} \int_{\Omega} \frac{p_{1}}{\bar{\rho}} \rho^{2}+|u|^{2}+\frac{p_{2}}{p_{3}} \theta^{2} d x+\int_{0}^{t} \int_{\Omega} \hat{\mu}|D u|^{2}+\left(\hat{\mu}+\hat{\mu}^{\prime}\right)\left(u_{x_{j}}^{j}\right)^{2}+\frac{p_{2}}{p_{3}} \hat{\kappa}|D \theta|^{2} d x d t \\
& =\frac{1}{2} \int \frac{p_{1}}{\bar{\rho}} \rho_{0}^{2}+\left|u_{0}\right|^{2}+\frac{p_{2}}{p_{3}} \theta_{0}^{2} d x+\int_{0}^{t} A_{0} d t,
\end{aligned}
$$

where $A_{0}$ is defined by (4.9). If we use the notation $d \rho / d t$ in (4.9), we can obtain (4.8), $l=0$ from this equality. The time derivative can be treated similarly, because it has the same boundary conditions. Next compute the integral

$$
\int_{0}^{t} \int_{\Omega} \rho_{t}\left(L^{0}-f^{0}\right)+u_{t}^{i}\left(L^{i}-f^{i}\right)+\theta_{t}\left(L^{4}-f^{4}\right) d x d t=0 .
$$

Integration by parts gives, by use of Schwarz inequality,

$$
\begin{aligned}
\int_{\Omega} \hat{\mu}|D u|^{2} & +\left(\hat{\mu}+\hat{\mu}^{\prime}\right)\left(u_{x_{J}}^{j}\right)^{2}+\hat{\kappa}|D \theta|^{2}+p_{1} \rho u_{x_{j}}^{j} d x+\frac{1}{2} \int_{0}^{t} \int_{\Omega} \rho_{t}^{2}+\left|u_{t}\right|^{2}+\theta_{t}^{2} d x d t \\
& \leqq \int_{\Omega} \hat{\mu}\left|D u_{0}\right|^{2}+\left(\hat{\mu}+\hat{\mu}^{\prime}\right)\left(u_{0, x_{j}}^{j}\right)^{2}+\hat{\kappa}\left|D \theta_{0}\right|^{2}+p_{1} \rho_{0} u_{0, x_{j}}^{j} d x \\
& +C \int_{0}^{t} \int_{\Omega}|D(u, \theta)(s)|^{2}+|\tilde{f}|^{2} d x d t,
\end{aligned}
$$

where $\tilde{f}=\left(f^{0}-u^{j} \rho_{x_{j}}, f^{1}, f^{2}, f^{3}, f^{4}\right)$. If we use Schwarz inequality for the term $\rho u_{x_{j}}^{j}$, we obtain (4.10), $l=0$. The estimate (4.10), $l=1$ is obtained similarly.

In the following we first treat the case of the half space $\Omega=R_{+}^{3}$ since it is easier to obtain estimates in this case than for the exterior domains treated later.

Since the tangential derivatives of the solution of (3.4) satisfy the same boundary conditions (3.5), we can obtain the estimates for these similarly to the above Lemma 4.4. Let us denote the tangential derivatives by $\partial=\left(\partial_{x_{1}}, \hat{\partial}_{x_{2}}\right)$ and integrate the equality for each $k=1,2,3$ by use of integration by parts

$$
\frac{p_{1}}{\bar{\rho}} \partial^{k}\left(L^{0}-f^{0}\right) \partial^{k} \rho+\partial^{k}\left(L^{i}-f^{i}\right) \partial^{k} u^{i}=0
$$

Thus we have

Lemma 4.5. For $k=1,2,3$,

$$
\begin{aligned}
& \left\|\partial^{k}(\rho, u)(t)\right\|^{2}+\int_{0}^{t}\left\|D \partial^{k} u(s)\right\|^{2}+\left\|\partial^{k} \frac{d \rho}{d t}(s)\right\|^{2} d s \\
& \leqq C\left\{\left\|\partial^{k}(\rho, u)(0)\right\|^{2}+\int_{0}^{t}\left\|\partial^{k} f^{0}(s)\right\|^{2}+\left\|\partial^{k-1} f(s)\right\|^{2}+\left|A_{k+1}\right| d s\right\},
\end{aligned}
$$


where

$$
A_{k+1}=\int_{\Omega} \frac{p_{1}}{\bar{\rho}} \partial^{k} \rho \partial^{k}\left(f^{0}-u^{j} \rho_{x_{J}}\right) d x \text { for each } \quad k=1,2,3 .
$$

Then we have to obtain the estimates for the normal derivatives of solution. To do that we use the following equations from (3.4).

$$
\begin{aligned}
& \left(\frac{d \rho}{d t}\right)_{x_{3}}+\bar{\rho} u_{x_{3} x_{3}}^{j}=f_{x_{3}}^{0}, \\
& u_{t}^{3}-\hat{\mu} \Delta u^{3}-\left(\hat{\mu}+\hat{\mu}^{\prime}\right) u_{x_{j} x_{3}}^{j}+p_{1} \rho_{x_{3}}+p_{2} \theta_{x_{3}}=f^{3} .
\end{aligned}
$$

If we eliminate the term $u_{x_{3} x_{3}}^{3}$ from these, we have

$$
\begin{aligned}
\frac{2 \hat{\mu}+\hat{\mu}^{\prime}}{\bar{\rho}}\left(\frac{d \rho}{d t}\right)_{x_{3}} & +p_{1} \rho_{x_{3}}=-u_{t}^{3}-p_{2} \theta_{x_{3}}+\frac{2 \hat{\mu}+\hat{\mu}^{\prime}}{\bar{\rho}} f_{x_{3}}^{0}+f^{3} \\
& +\hat{\mu}\left(u_{x_{1} x_{1}}^{3}+u_{x_{2} x_{2}}^{3}\right)+\hat{\mu}^{\prime}\left(u_{x_{1}}^{1}+u_{x_{2}}^{2}\right)_{x_{3}},
\end{aligned}
$$

where we note the second derivatives of $u$ at the last two terms on the right hand side contain one tangential derivative. Multiply (4.14) by $\rho_{x_{3}}$ and $(d \rho / d t)_{x_{3}}$ respectively and integrate them respectively. We obtain after integration by parts

$$
\begin{aligned}
& \int \frac{2 \hat{\mu}+\hat{\mu}^{\prime}}{2 \bar{\rho}} \rho_{x_{3}}^{2} d x+\int_{0}^{t} \int p_{1} \rho_{x_{3}}^{2} d x d t \\
& =\int \frac{2 \hat{\mu}+\hat{\mu}^{\prime}}{2 \bar{\rho}} \rho_{0, x_{3}}^{2} d x+\int_{0}^{t} \int \frac{2 \hat{\mu}+\hat{\mu}^{\prime}}{2 \bar{\rho}}\left(-u_{x_{j}}^{j} \rho_{x_{3}}+2 u_{x_{3}}^{j} \rho_{x_{3}}\right) \rho_{x_{3}} d x d t \\
& \quad+\int_{0}^{t} \int\left\{-u_{t}^{3}-p_{2} \theta_{x_{3}}+\hat{\mu}\left(u_{x_{1} x_{1}}^{3}+u_{x_{2} x_{2}}^{3}+u_{x_{1} x_{3}}^{1}\right.\right. \\
& \left.\left.\quad+u_{x_{2} x_{3}}^{2}\right)\right\} \rho_{x_{3}}+\left(\frac{2 \hat{\mu}+\hat{\mu}^{\prime}}{\bar{\rho}} f_{x_{3}}^{0}+f^{3}\right) \rho_{x_{3}} d x d t \\
& \leqq \\
& p_{1} \int_{0}^{t} \int_{0}^{t} \rho_{x_{3}}^{2} d x d t+C\left\{\|D \rho(0)\|^{2}+\int_{0}^{t}\left|B_{0,0}\right| d t\right. \\
& \left.\quad+\int_{0}^{t}\left\|u_{t}\right\|^{2}+\|D \partial u\|^{2}+\|D \theta\|^{2}+\left\|D f^{0}\right\|^{2}+\left\|f^{3}\right\|^{2} d s\right\},
\end{aligned}
$$

and

$$
\begin{aligned}
& \int_{0}^{t} \int_{0} \frac{2 \hat{\mu}+\hat{\mu}^{\prime}}{\bar{\rho}}\left\{\left(\frac{d \rho}{d t}\right)_{x_{3}}\right\}^{2} d x d t+\frac{p_{1}}{2} \int \rho_{x_{3}}^{2} d x \\
& =\frac{p_{1}}{2} \int_{0, x_{3}}^{2} d x+\int_{0}^{t} \int \frac{p_{1}}{2}\left(-u_{x_{3}}^{j} \rho_{x_{3}}+u_{x_{3}}^{j} \rho_{x_{3}}\right) \rho_{x_{3}} d x d t \\
& \quad+\int_{0}^{t} \int \frac{2 \hat{\mu}+\hat{\mu}^{\prime}}{\bar{\rho}}\left(\frac{d \rho}{d t}\right)_{x_{3}}\left\{-u_{t}^{3}-p_{2} \theta_{x_{3}}+\hat{\mu}\left(u_{x_{1} x_{1}}^{3}+u_{x_{2} x_{2}}^{3}\right.\right.
\end{aligned}
$$




$$
\begin{aligned}
& \left.\left.+u_{x_{1} x_{3}}^{1}+u_{x_{2} x_{3}}^{2}\right)+\frac{2 \hat{\mu}+\hat{\mu}^{\prime}}{\bar{\rho}} f_{x_{3}}^{0}+f^{3}\right\} d x d t \\
\leqq & \frac{2 \hat{\mu}+\hat{\mu}^{\prime}}{2 \bar{\rho}} \int_{0}^{t} \int\left(\frac{d \rho}{d t}\right)_{x_{3}}^{2} d x d t+C\left\{\|D \rho(0)\|^{2}+\int_{0}^{t}\left|B_{0,0}\right| d t\right. \\
& \left.+\int_{0}^{t}\left\|u_{t}\right\|^{2}+\|D \theta\|^{2}+\|D \partial u\|^{2}+\left\|D f^{0}\right\|^{2}+\left\|f^{3}\right\|^{2} d x d t\right\}
\end{aligned}
$$

respectively. Thus we have obtained the following

Lemma 4.6. For $k+l=0,1,2$,

$$
\begin{aligned}
& \left\|\partial^{k} \partial_{3}^{l+1} \rho(t)\right\|^{2}+\int_{0}^{t}\left\|\partial^{k} \partial_{3}^{l+1} \rho(s)\right\|^{2}+\left\|\partial^{k} \partial_{3}^{l+1}\left(\frac{d \rho}{d t}\right)(s)\right\|^{2} d s \\
& \leqq C\left\{\|D \rho(0)\|_{k+l}^{2}+\int_{0}^{t}\left\|\partial^{k+1} \partial_{3}^{l} D u\right\|^{2}+\left\|\partial^{k} \partial_{3}^{l} u_{t}\right\|^{2}\right. \\
& \left.\quad+\|D(u, \theta)(s)\|_{k+l}^{2}+\left\|f^{0}\right\|_{k+l+1}^{2}+\|f\|_{k+l}^{2}+\left|B_{k, l}\right| d s\right\},
\end{aligned}
$$

where

$$
B_{k, l} \equiv \int\left\{\partial^{k} \partial_{3}^{l}\left(\frac{d \rho}{d t}\right)_{x_{3}}-\partial^{k} \partial_{3}^{l} \rho_{t x_{3}}\right\} \partial^{k} \partial_{3}^{l} \rho_{x_{3}} d x,
$$

and here the summation is not taken for $k$ and $l$.

Proof. For $k+l=1$ and 2 we differentiate Eq. (4.14) by $\partial^{k} \partial_{3}^{l}$ and multiply it by $\partial^{k} \partial_{3}^{l+1} \rho$ and $\partial^{k} \partial_{3}^{l+1}(d \rho / d t)$ respectively. Integration by parts gives (4.15) and (4.16), in this case similarly to that for $k=l=0$.

Last we use Lemma 4.3 for stokes equation (4.6) with $\left.u\right|_{\partial \Omega}=0$, where $h$ and $g^{i}$ have the following explicit forms.

$$
\begin{aligned}
& h=f^{0}-\frac{d \rho}{d t} \\
& g^{i}=-u_{t}^{i}+\frac{\hat{\mu}+\hat{\mu}^{\prime}}{\bar{\rho}} h_{x_{\mathrm{t}}}-p_{2} \theta_{x_{\mathrm{t}}}+f^{i}, \quad i=1,2,3 .
\end{aligned}
$$

Lemma 4.7. For $k+l=0,1,2$, we have

$$
\begin{aligned}
& \left\|D^{2+l} \partial^{k} u\right\|+\left\|D^{1+l} \partial^{k} \rho\right\| \leqq C\left\{\left\|u_{t}\right\|_{k+l}+\left\|\partial^{k}\left(\frac{d \rho}{d t}\right)\right\|_{1+l}\right. \\
& \left.+\|D \theta\|+\left\|f^{0}\right\|_{1+k+l}+\|f\|_{k+l}\right\} .
\end{aligned}
$$

Now we can combine the above Lemmas $4.1-4.7$ to obtain necessary a priori estimates. First we obtain the $H^{2}$ version of norm $N(0, t)$, i.e., 


$$
\begin{aligned}
\|\rho, u, \theta(t)\|_{2}^{2}+\left\|\rho_{t}(t)\right\|_{1}^{2}+\left\|u_{t}, \theta_{t}(t)\right\|^{2} & \\
& +\int_{0}^{t}\left\|\rho_{t}(s), D \rho(s)\right\|_{1}^{2}+\left\|u_{t}, \theta_{t}(s)\right\|_{1}^{2}+\|D(u, \theta)(s)\|_{2}^{2}+\left\|\frac{d \rho}{d t}(s)\right\|_{2}^{2} d s \\
\leqq & C\left\{\|\rho, u, \theta(0)\|_{2}^{2}+\sup _{0 \leqq s \leqq t}\left\{\left\|f^{0}-u^{j} \rho_{x_{j}}\right\|_{1}^{2},\|f(s)\|^{2}\right\}\right. \\
& \quad+\int_{0}^{t}\left\|f^{0}\right\|_{2}^{2}+\left\|f_{t}^{0}\right\|^{2}+\|f\|_{1}^{2}+\left\|u^{j} \rho_{x_{j}}\right\|_{1}^{2}+\sum_{k=0}^{3}\left|A_{k}\right|+\sum_{k+l=0}^{1}\left|B_{k, l}\right| d s .
\end{aligned}
$$

It is proved by fifteen steps as follows:

(i) By Lemma $4.4, l=0$, we have

$$
\begin{aligned}
\|\rho, u, \theta(t)\|^{2}+\int_{0}^{t}\|D(u, \theta)(s)\|^{2}+\left\|\frac{d \rho}{d t}(s)\right\|^{2} d s \leqq & C\left\{\|\rho, u, \theta(0)\|^{2}+\int_{0}^{t}\left\|f^{0}(s)\right\|^{2}\right. \\
& \left.+\left|A_{0}\right| d s\right\}
\end{aligned}
$$

(ii) By Lemma 4.4, $k=0$, and (4.20), we have

$$
\begin{aligned}
& \|D(u, \theta)(t)\|^{2}+\int_{0}^{t}\left\|\rho_{t}, u_{t}, \theta_{t}(s)\right\|^{2} d s \\
& \leqq \cdots+C\left\{\|D(u, \theta)(0)\|^{2}+\|\rho(0)\|^{2}+\int_{0}^{t}\|\tilde{f}(s)\|^{2} d s\right\} .
\end{aligned}
$$

where ... means the terms already appeared on the right hand side of inequalities (4.20)-(4.32), especially (4.20) for (4.21).

(iii) By Lemma $4.4, l=1$, we have

$$
\begin{gathered}
\left\|\rho_{t}, u_{t}, \theta_{t}(t)\right\|^{2}+\int_{0}^{t}\left\|D\left(u_{t}, \theta_{t}\right)(s)\right\|^{2}+\left\|\left(\frac{d \rho}{d t}\right)(s)\right\|_{t}^{2} d s \\
\leqq C\left\{\left\|\rho_{t}, u_{t}, \theta_{t}(0)\right\|^{2}+\int_{0}^{t}\left\|f_{t}^{0}(s)\right\|^{2}+\left|A_{1}\right| d s\right\} .
\end{gathered}
$$

(iv) By Lemma 4.2 for $\theta$ and by (4.20)-(4.22), we have

$$
\begin{aligned}
\left\|D^{2} \theta(t)\right\|^{2} & +\int_{0}^{t}\left\|D^{2} \theta(s)\right\|^{2} d s \leqq \cdots+C\left\{\left\|f^{4}(t)\right\|^{2}\right. \\
& \left.+\int_{0}^{t}\left\|f^{4}(s)\right\|^{2} d s\right\} .
\end{aligned}
$$

(v) We have the estimate of tangential derivatives of $\rho$ by Lemma $4.5, k=1$,

$$
\begin{aligned}
\|\partial \rho(t)\|^{2} & +\int_{0}^{t}\|D \partial u(s)\|^{2}+\left\|\partial\left(\frac{d \rho}{d t}\right)(s)\right\|^{2} d s \leqq \cdots+\|\partial \rho(0)\|^{2} \\
& +C \int_{0}^{t}\left\|\partial f^{0}(s)\right\|^{2}+\|f(s)\|^{2}+\left|A_{2}\right| d s .
\end{aligned}
$$

(vi) The estimate of the normal derivative for $\rho$ and $d \rho / d t$ follows from Lemma 
4.6, $k+l=0$, and from (4.24), (4.21) and (4.20)

$$
\begin{aligned}
& \left\|\partial_{3} \rho(t)\right\|^{2}+\int_{0}^{t}\left\|\partial_{3} \rho(s)\right\|^{2}+\left\|\partial_{3} \frac{d \rho}{d t}(s)\right\|^{2} d s \\
& \leqq \cdots+C\left\{\left\|\partial_{3} \rho(0)\right\|^{2}+\int_{0}^{t}\left\|f^{0}\right\|_{1}^{2}+\|f\|^{2}+\left|B_{0,0}\right| d s\right\} .
\end{aligned}
$$

(vii) Then we have the second derivative of $u$ by Lemma 4.2 for $u$ and $k=2$, and by (4.19), (4.21), (4.22), (4.24), (4.25).

$$
\left\|D^{2} u(t)\right\|^{2} \leqq \cdots+C\|f(t)\|^{2} .
$$

(viii) Further since $d \rho / d t \in L_{2}\left(0, t ; H^{1}\right)$, we have by Lemma $4.7, k+l=0$, and by (4.21), (4.20), (4.24) and (4.25),

$$
\int_{0}^{t}\left\|D^{2} u(s)\right\|^{2}+\|D \rho(s)\|^{2} d s \leqq \cdots+\int_{0}^{t}\left\|f^{0}\right\|_{1}^{2}+\|f\|^{2} d s .
$$

(ix) By Lemma 4.2 for $\theta, k=3$ and by (4.27), (4.21) and (4.22), we have

$$
\int_{0}^{t}\left\|D^{3} \theta(s)\right\|^{2} d s \leqq \cdots+\int_{0}^{t}\left\|f^{4}(s)\right\|_{1}^{2} d s .
$$

(x) By Lemma 4.5, $k=2$ we have

$$
\begin{aligned}
& \left\|\partial^{2} \rho(t)\right\|^{2}+\int_{0}^{t}\left\|D \partial^{2} u(s)\right\|^{2}+\left\|\partial^{2} \frac{d \rho}{d t}(s)\right\|^{2} d s \\
& \quad \leqq C\left\{\left\|\partial^{2}(\rho, u)(0)\right\|^{2}+\int_{0}^{t}\left\|\partial^{2} f^{0}(s)\right\|^{2}+\|\partial f(s)\|^{2}+\left|A_{3}\right| d s\right\} .
\end{aligned}
$$

(xi) By Lemma 4.6, $k=1, l=0$ and by (4.29), (4.22), we have

$$
\begin{aligned}
& \left\|\partial \partial_{3} \rho(t)\right\|^{2}+\int_{0}^{t}\left\|\partial \partial_{3} \rho(s)\right\|^{2}+\left\|\partial \partial_{3} \frac{d \rho}{d t}(s)\right\|^{2} d s \\
& \quad \leqq \cdots+C\left\{\left\|\partial \partial_{3} \rho(0)\right\|^{2}+\int_{0}^{t}\left\|f^{0}(s)\right\|_{2}^{2}+\|f(s)\|_{1}^{2}+\left|B_{1,0}\right| d s\right\} .
\end{aligned}
$$

(xii) By Lemma 4.7, $k=1, l=0$ and by (4.29), (4.30) and (4.22), we have

$$
\int_{0}^{t}\left\|D^{2} \partial u(s)\right\|^{2}+\|D \partial \rho(s)\|^{2} d s \leqq \cdots+\int_{0}^{t}\left\|f^{0}(s)\right\|_{2}^{2}+\|f(s)\|_{1}^{2} d s .
$$

(xiii) By Lemma 4.6, $k=0, l=1$ and by (4.31), (4.22), we have

$$
\begin{gathered}
\left\|\partial_{3}^{2} \rho(t)\right\|^{2}+\int_{0}^{t}\left\|\partial_{3}^{2} \rho(s)\right\|^{2}+\left\|\partial_{3}^{2} \frac{d \rho}{d t}(s)\right\|^{2} d s \\
\leqq \cdots+C\left\{\|\rho(0)\|_{2}^{2}+\int_{0}^{t}\left|B_{0,1}\right| d s\right\} .
\end{gathered}
$$

(xiv) By Lemma 4.7, $k=0, l=1$ and by (4.29), (4.30), (4.32) and (4.22), we have

$$
\int_{0}^{t}\left\|D^{3} u(s)\right\|^{2}+\left\|D^{2} \rho(s)\right\|^{2} d s \leqq \cdots
$$


(xv) Then by the Eq. (3.4)

$$
\begin{aligned}
\left\|\rho_{t}(t)\right\|_{1}^{2}+\int_{0}^{t}\left\|\rho_{t}(s)\right\|_{1}^{2} d s \leqq & \cdots+\left\|f^{0}-u^{j} \rho_{x_{j}}\right\|_{1}^{2} \\
& +\int_{0}^{t}\left\|f^{0}-u^{j} \rho_{x_{j}}(s)\right\|_{1}^{2} d s .
\end{aligned}
$$

Thus if we sum (4.20)-(4.34), we arrive at the $H^{2}$ energy estimate (4.19).

To elevate the differentiability once to obtain the estimate of norm $N(0, t)$, we can repeat the above argument beginning from Lemma 4.4, $k=1$ and by use of Lemma 4.2, $k=3$, Lemma 4.5, $k=3$, Lemma 4.6, $k+l=2$ and Lemma $4.7, k+l=2$. Therefore we arrive at the estimate for $N(0, t)$.

$$
\begin{aligned}
N(0, t)^{2} \equiv & \|\rho, u, \theta(t)\|_{3}^{2}+\left\|\rho_{t}(t)\right\|_{2}^{2}+\left\|u_{t}, \theta_{t}(t)\right\|_{1}^{2} \\
& +\int_{0}^{t}\left\|\rho_{t}, D \rho(s)\right\|_{2}^{2}+\left\|u_{t}, \theta_{t}(s)\right\|_{2}^{2}+\|D(u, \theta)(s)\|_{3}^{2} d s \\
\leqq & C\left\{\|\rho, u, \theta(0)\|_{3}^{2}+\sup _{0 \leqq s \leqq t}\left\{\left\|f^{0}-u^{j} \rho_{x_{j}}(s)\right\|_{2}^{2}+\|f(s)\|_{1}^{2}\right\}\right. \\
& +\int_{0}^{t}\left\|f^{0}(s)\right\|_{3}^{2}+\|f(s)\|_{2}^{2}+\left\|f_{t}(s)\right\|^{2}+\left\|\left(u^{j} \rho_{x_{j}}\right)_{t}\right\|^{2}+\left\|u^{j} \rho_{x_{j}}\right\|_{2}^{2} \\
& \left.+\sum_{k=0}^{4}\left|A_{k}\right|+\sum_{k+l=0}^{2}\left|B_{k, l}\right| d s\right\} .
\end{aligned}
$$

Last we have to show

\section{Lemma 4.8.}

$$
\begin{aligned}
& \sup _{0 \leqq s \leqq t}\left\{\left\|f^{0}-u^{j} \rho_{x_{j}}(s)\right\|_{2}^{2}+\|f(s)\|_{1}^{2}\right\}+\int_{0}^{t}\left\|f^{0}(s)\right\|_{3}^{2}+\left\|\left(f^{0}-u^{j} \rho_{x_{j}}\right)_{t}(s)\right\|^{2} \\
& \quad+\left\|f_{t}(s)\right\|^{2}+\|\tilde{f}(s)\|_{2}^{2}+\sum_{k=0}^{4}\left|A_{k}\right|+\sum_{k+l=0}^{2}\left|B_{k, l}\right| d s \\
& \quad \leqq C_{2}\left(N(0, t)+\|\Phi\|_{5}\right) N^{2}(0, t) .
\end{aligned}
$$

It is proved by use of Lemma 4.1 and integration by parts. We show only the term $A_{0}$ and omit the proof of the other terms which can be treated similarly. Let us recall (4.9) and compute the following

$$
\begin{aligned}
& \left|\int \rho\left(f^{0}-u^{j} \rho_{x_{j}}\right) d x\right|=\left|\int \dot{\rho}\left\{(\bar{\rho}-\tilde{\rho}) u^{j}-\rho u^{j}\right\}_{x_{j}} d x\right| \\
& \quad=\left|\int \rho_{x_{j}}\left\{(\bar{\rho}-\tilde{\rho}) u^{j}-\rho u^{j}\right\} d x\right| \\
& \quad \leqq\|D \rho\|\left\{\left(\int(\tilde{\rho}-\tilde{\rho})^{2}|u|^{2} d x\right)^{1 / 2}+\left(\int \rho^{2}|u|^{2} d x\right)^{1 / 2}\right\} \\
& \quad \leqq\|D \rho\|\left\{\|\bar{\rho}-\tilde{\rho}\|_{L_{3}}\|u\|_{L_{6}}+\|\rho\|_{L_{3}}\|u\|_{L_{6}}\right\} \\
& \quad \leqq C\|D \rho\|\|D u\|\left\{\|\Phi\|_{3}+\|\rho\|_{1}\right\} \leqq C N(0, t)^{2}\left\{\|\Phi\|_{3}+N(0, t)\right\}
\end{aligned}
$$




$$
\begin{aligned}
& \left|\int u^{i}\left\{\left(\frac{\mu}{\rho+\tilde{\rho}}-\hat{\mu}\right) u_{x_{\jmath} x_{\jmath}}^{i}+\frac{1}{\rho+\tilde{\rho}} \mu_{x_{\jmath}} u_{x_{\jmath}}^{i}\right\} d x\right| \\
& \quad \leqq \int\left|u_{x_{\jmath}}^{i}\left(\frac{\mu}{\rho+\tilde{\rho}}-\hat{\mu}\right) u_{x_{J}}^{i}\right|+\left|\hat{\mu} u^{i}\left(\frac{1}{\rho+\tilde{\rho}}\right)_{x_{j}} u_{x_{j}}^{i}\right| d x \\
& \quad \leqq\left|\frac{\mu}{\rho+\tilde{\rho}}-\hat{\mu}\right|_{0}\|D u\|^{2}+\left\|\left(\frac{1}{\rho+\tilde{\rho}}\right)_{x_{\jmath}}\right\|_{L_{3}}\|u\|_{L_{6}}\|D u\| \\
& \leqq C N(0, t)^{2}\left\{\|\Phi\|_{3}+N(0, t)\right\} .
\end{aligned}
$$

The remaining terms in $A_{0}$ can be treated in the same way as above.

Finally let us turn to the case of exterior domains. In this case, since we cannot generally designate a coordinate system over all of $\Omega$ so that directions are consistent with the normal and tangential directions on the boundary $\partial \Omega$ as in the case of the half space, we have to modify the Lemmas 4.5 through 4.7. To do that we shall separate the estimates of the solution into that over the region away from the boundary $\partial \Omega$ and that near the boundary $\partial \Omega$. Let $\chi_{0}(x)$ be any fixed cut-off function in $\mathscr{C}^{\infty}(\Omega)$ such that support $\chi_{0} \subset \Omega$ and $\chi_{0} \equiv 1$ outside of a bounded region $\Omega^{\prime}$. Then we have the following as the estimate on the region away from the boundary.

Lemma 4.9. For $k=1,2,3$, it holds that

$$
\begin{aligned}
& \left\|\chi_{0} D^{k} \rho(t)\right\|^{2}+\int_{0}^{t}\left\|\chi_{0} D^{k} \rho(s)\right\|^{2} d s \leqq C\left\{\left\|D^{k} \rho(0)\right\|^{2}+\left\|D^{k-1} u(0)\right\|^{2}\right. \\
& +\left\|D^{k-1} u(t)\right\|^{2}+\int_{0}^{t}\left\|f^{0}-u^{j} \rho_{x_{j}}\right\|_{k-1}^{2}+\|f\|_{k-1}^{2} \\
& \left.+\|D(u, \theta)\|_{k-1}^{2}+\left|A_{k+1}^{\prime}\right| d s\right\}, \\
& \left\|\chi_{0} D^{k}(\rho, u)(t)\right\|^{2}+\int_{0}^{t}\left\|\chi_{0} D^{k+1} u(s)\right\|^{2}+\left\|\chi_{0} D^{k} \frac{d \rho}{d t}(s)\right\|^{2} d s \\
& \leqq C\left\{\left\|D^{k}(\rho, u)(0)\right\|^{2}+\int_{0}^{t}\left\|D^{k-1} f\right\|^{2}+\left\|D^{k} f^{0}\right\|^{2}\right. \\
& \left.+\left\|\chi_{0} D^{k} \rho\right\|^{2}+\|D(u, \theta)\|_{k-1}^{2}+\left|A_{k+1}^{\prime}\right| d s\right\},
\end{aligned}
$$

where

$$
A_{k+1}^{\prime}=\int_{\Omega} \frac{p_{1}}{\bar{\rho}} \chi_{0}^{2}\left(D^{k} \rho\right) D^{k}\left(f^{0}-u^{j} \rho_{x_{j}}\right) d x
$$

and here the summation is not taken for $k$.

Proof. Compute the integral

$$
\int_{0}^{t} \int_{\Omega} \frac{2 \hat{\mu}+\hat{\mu}^{\prime}}{\bar{\rho}} \chi_{0}^{2}\left(L^{0}-f^{0}\right)_{x_{1}} \rho_{x_{1}}+\chi_{0}^{2}\left(L^{\imath}-f^{\imath}\right) \rho_{x_{1}} d x d s=0 .
$$


After integration by parts, we have

$$
\begin{aligned}
& \int_{\Omega} \frac{2 \hat{\mu}+\hat{\mu}^{\prime}}{2 \bar{\rho}} \chi_{0}^{2}|D \rho|^{2}+\left.\chi_{0}^{2} u^{i} \rho_{x_{l}} d x\right|_{0} ^{t}+\int_{0}^{t} \int_{\Omega} p_{1} \chi_{0}^{2}|D \rho|^{2} d x d s \\
& =\int_{0}^{t} \int_{\Omega}-2 \hat{\mu} \chi_{0} \chi_{0, x_{J}} u_{x_{J}}^{i} \rho_{x_{i}}+2 \hat{\mu} \chi_{0} \chi_{0, x_{1}} u_{x_{j}}^{i} \rho_{x_{J}}+\chi_{0}^{2} f^{i} \rho_{x_{1}} \\
& \quad \quad-p_{2} \chi_{0}^{2} \rho_{x_{l}} \theta_{x_{1}}+\left(2 \chi_{0} \chi_{0, x_{l}} u^{i}+\chi_{0}^{2} u_{x_{J}}^{j}\right)\left(u^{j} \rho_{x_{J}}-f^{0}+\bar{\rho} u_{x_{J}}^{j}\right) d x+A_{2}^{\prime} d s .
\end{aligned}
$$

Since the support of $D \chi_{0}$ is contained in $\Omega^{\prime}$, we can make use of (4.2) and consequently the Schwarz inequality gives (4.37), $k=1$. The other cases $k=2,3$, are obtained similarly. To prove (4.38), compute the integral

$$
\int_{0}^{t} \int_{\Omega} \chi_{0}^{2} \frac{p_{1}}{\bar{\rho}}(D \rho) D\left(L^{0}-f^{0}\right)+\chi_{0}^{2}\left(D u^{i}\right) D\left(L^{i}-f^{i}\right) d x d s=0 .
$$

Integration by parts gives

$$
\begin{aligned}
\frac{1}{2} \int_{\Omega} \chi_{0}^{2} & \frac{p_{1}}{\bar{\rho}}|D \rho|^{2}+\left.\chi_{0}^{2}|D u|^{2} d x\right|_{0} ^{t}+\int_{0}^{t} \int_{\Omega} \chi_{0}^{2} \hat{\mu}\left|D^{2} u\right|^{2}+\chi_{0}^{2}\left(\hat{\mu}+\hat{\mu}^{\prime}\right)\left|D u_{x_{J}}^{j}\right|^{2} d x d s \\
= & \int_{0}^{t} \int_{\Omega} \chi_{0}^{2} p_{2}\left(D u_{x_{l}}^{i}\right) D \theta+2 \chi_{0} \chi_{0, x_{i}} p_{2}\left(D u^{i}\right) D \theta+2 \chi_{0} \chi_{0, x_{i}} p_{1}\left(D u^{i}\right) D \rho-\chi_{0}^{2} f^{i} u_{x_{J} x_{J}}^{i} \\
& \quad-2 \chi_{0} \chi_{0, x_{J}} f^{i} u_{x_{J}}^{i} d x+A_{2}^{\prime} d s,
\end{aligned}
$$

which implies (4.38), $k=1$ after using the Schwarz inequality. The cases $k=2,3$ are obtained similarly.

Next let us establish the estimates near the boundary. To do that we choose a finite number of bounded open sets $\left\{\mathcal{O}_{j}\right\}_{j=1}^{N}$ in $R^{3}$ such that

$$
\bigcup_{j=1}^{N} \mathcal{O}_{j} \supset \partial \Omega
$$

and in each set $\mathcal{O}_{j}$ we choose local coordinates $(\psi, \phi, r)$ as follows;

(i) the boundary $\mathcal{O}_{j} \cap \partial \Omega$ is the image of smooth functions $y^{i}=y^{i}(\psi, \phi)$ satisfying (e.g., take the local geodesic polar coordinate)

$$
\left|y_{\psi}\right|=1, \quad y_{\psi}^{i} y_{\phi}^{i}=0, \quad\left|y_{\phi}\right| \geqq \delta>0,
$$

where $\delta$ is some positive constant independent of $j=1, \ldots, N$.

(ii) any $x$ in $\mathcal{O}_{j}$ is represented by

$$
x^{i}=x^{i}(\psi, \phi, r)=r n^{i}(\psi, \phi)+y^{i}(\psi, \phi),
$$

where $n^{i}(\psi, \phi)$ represents the external unit normal vector at the point of the boundary coordinated $(\psi, \phi)$. Here and in what follows we omit the suffix $j$ for simplicity. Let us define the unit vectors $e_{1}^{i}$ and $e_{2}^{i}$ by $e_{1}^{i}=y_{\psi}^{i}$ and $e_{2}^{i}=y_{\phi}^{i} /\left|y_{\phi}\right|$. Then Frenet-Serret's formula gives that there exist smooth functions $\left(\alpha, \beta, \gamma, \alpha^{\prime}, \beta^{\prime}, \gamma^{\prime}\right)$ of $(\psi, \phi)$ satisfying 


$$
\begin{gathered}
\frac{\partial}{\partial \psi}\left(\begin{array}{l}
e_{1} \\
e_{2} \\
n
\end{array}\right)^{i}=\left(\begin{array}{rrr}
0 & -\gamma & -\alpha \\
\gamma & 0 & -\beta \\
\alpha & \beta & 0
\end{array}\right)\left(\begin{array}{l}
e_{1} \\
e_{2} \\
n
\end{array}\right)^{i}, \\
\frac{\partial}{\partial \phi}\left(\begin{array}{l}
e_{1} \\
e_{2} \\
n
\end{array}\right)^{\prime}=\left(\begin{array}{rrr}
0 & -\gamma^{\prime} & -\alpha^{\prime} \\
\gamma^{\prime} & 0 & -\beta^{\prime} \\
\alpha^{\prime} & \beta^{\prime} & 0
\end{array}\right)\left(\begin{array}{l}
e_{1} \\
e_{2} \\
n
\end{array}\right)^{i} .
\end{gathered}
$$

An elementary calculation shows that the Jacobian $J$ of the transformation (4.40) is given by

$$
J=\left|x_{\psi} \times x_{\phi}\right|=\left|y_{\phi}\right|+\left(\alpha\left|y_{\phi}\right|+\beta^{\prime}\right) r+\left(\alpha \beta^{\prime}-\beta \alpha^{\prime}\right) r^{2} .
$$

By (4.41) we can see the transformation (4.40) is regular choosing $r$ small if needed. Therefore the functions $(\psi, \phi, r)_{x_{1}}(x)$ make sense and is calculated as

$$
\begin{aligned}
\psi_{x_{i}} & =\frac{1}{J}\left(x_{\phi} \times x_{r}\right)_{i}=\frac{1}{J}\left(A e_{1}^{i}+B e_{2}^{i}\right), \\
\phi_{x_{i}} & =\frac{1}{J}\left(x_{r} \times x_{\phi}\right)_{i}=\frac{1}{J}\left(C e_{1}^{i}+D e_{2}^{i}\right), \\
r_{x_{i}} & =\frac{1}{J}\left(x_{\psi} \times x_{\phi}\right)_{i}=n_{i},
\end{aligned}
$$

where $A=\left|y_{\phi}\right|+\beta^{\prime} r, B=-r \alpha^{\prime}, C=-\beta r, D=1+\alpha r$ and $J=A D-B C>0$. Hence (4.42) implies

$$
\partial_{x_{i}}=\frac{1}{J}\left(A e_{1}^{i}+B e_{2}^{i}\right) \partial_{\psi}+\frac{1}{J}\left(C e_{1}^{i}+D e_{2}^{i}\right) \partial_{\phi}+n^{i} \partial_{r}
$$

Thus in each $\mathcal{O}_{j}$ we can rewrite the equations $\left\{(3.4)^{i}\right\}_{i=0}^{3}$ in the local coordinates $(\psi, \phi, r)$ as follows:

$$
\begin{aligned}
L^{0}= & \frac{d \rho}{d t}+\frac{\bar{\rho}}{J}\left(\left(A e_{1}^{i}+B e_{2}^{i}\right) u_{\psi}^{i}+\left(C e_{1}^{i}+D e_{2}^{i}\right) u_{\phi}^{i}+J n^{i} u_{r}^{i}\right)=f^{0}, \\
L^{i}= & u_{t}^{i}+\frac{\hat{\mu}}{J^{2}}\left(\left(A^{2}+B^{2}\right) u_{\psi \psi}^{i}+2(A C+B D) u_{\phi \psi}^{i}+\left(C^{2}+D^{2}\right) u_{\phi \phi}^{i}+J^{2} u_{r r}^{i}\right) \\
& + \text { first order terms of } u \text { and } \theta \\
& -\frac{1}{J}\left(A e_{1}^{i}+B e_{2}^{i}\right)\left(\frac{\hat{\mu}+\hat{\mu}^{\prime}}{\bar{\rho}} \frac{d \rho}{d t}+p_{1} \rho\right)_{\psi}+\frac{1}{J}\left(C e_{1}^{i}+D e_{2}^{i}\right)\left(\frac{\hat{\mu}+\hat{\mu}^{\prime}}{\bar{\rho}} \frac{d \rho}{d t}+p_{1} \rho\right)_{\phi} \\
& +n^{i}\left(\frac{\hat{\mu}+\hat{\mu}^{\prime}}{\bar{\rho}} \frac{d \rho}{d t}+p_{1} \rho\right)_{r}+\frac{\hat{\mu}+\hat{\mu}^{\prime}}{\bar{\rho}} f_{x_{i}}^{0}=f^{i},
\end{aligned}
$$


where we note that $J^{2}=(A C+B D)^{2}-\left(A^{2}+B^{2}\right)\left(C^{2}+D^{2}\right)$. Let us denote the tangential derivatives by $\partial=\left(\partial_{\psi}, \partial_{\phi}\right)$ as before, and let $\chi_{j}(1 \leqq j \leqq N)$ be any fixed cutoff function in $\mathscr{C}_{0}^{\infty}\left(\mathcal{O}_{j}\right)$. Estimating the integral for $k=1,2,3$,

$$
\int_{0}^{t} \int_{\Omega} \chi_{j}^{2} \frac{p_{1}}{\bar{\rho}}\left(\partial^{k} \rho\right) \partial^{k}\left(L^{0}-f^{0}\right)+\chi_{j}^{2}\left(\partial^{k} u^{i}\right) \partial^{k}\left(L^{i}-f^{i}\right) J d \phi d \psi d r d s=0
$$

in the similar way as in Lemmas 4.5 and 4.9 , we can get

Lemma 4.10. For any positive $\varepsilon$ and $k=1,2,3$, it holds that

$$
\begin{aligned}
& \left\|\chi_{j} \partial^{k}(\rho, u)(t)\right\|^{2}+\int_{0}^{t}\left\|\chi_{j} D \partial^{k} u(s)\right\|^{2}+\left\|\chi_{j} \partial^{k} \frac{d \rho}{d t}(s)\right\|^{2} d s \\
& \leqq C\left\{\left\|\chi_{j} \partial^{k}(\rho, u)(0)\right\|^{2}+\int_{0}^{t} \varepsilon\|D \rho\|_{k-1}^{2}+\left(1+\varepsilon^{-1}\right)\|D(u, \theta)\|_{k-1}^{2}\right. \\
& \left.\quad+\left\|f^{0}\right\|_{k}^{2}+\|f\|_{k-1}^{2}+\left|A_{j, k+1}\right| d s\right\},
\end{aligned}
$$

where $A_{j, k+1}=\int_{\Omega} \frac{p_{1}}{\bar{\rho}} \chi_{j}^{2}\left(\partial^{k} \rho\right) \partial^{k}\left(f^{0}-u^{j} \rho_{x_{j}}\right) d x$, and here the summation is not taken for $k$.

In order to estimate the normal derivatives we make use of the equation $\partial_{r}\left(L^{0}-f^{0}\right)=0$ and $n^{i}\left(L^{i}-f^{i}\right)=0$, which have the form

$$
\begin{aligned}
\left(\frac{d \rho}{d t}\right)_{r} & +\frac{\bar{\rho}}{J}\left\{\left(A e_{1}^{i}+B e_{2}^{i}\right) u_{r \psi}^{i}+\left(C e_{1}^{i}+D e_{2}^{i}\right) u_{r \phi}^{i}+J n^{i} u_{r r}^{i}\right\} \\
& + \text { first order terms of } u=f_{r}^{0}, \\
n^{i} u_{t}^{i} & +\frac{\hat{\mu}}{J^{2}}\left\{\left(A^{2}+B^{2}\right) n^{i} u_{\psi \psi}^{i}+2(A C+B D) u_{\psi \phi}^{i}+\left(C^{2}+D^{2}\right) n^{i} u_{\phi \phi}^{i}+J^{2} n^{i} u_{r r}^{i}\right\} \\
& + \text { first order terms of } u \text { and } \theta \\
& +\frac{\hat{\mu}+\hat{\mu}^{\prime}}{\bar{\rho}}\left(\frac{d \rho}{d t}\right)_{r}+p_{1} \rho_{r}=n^{i} f^{i}+\frac{\hat{\mu}+\hat{\mu}^{\prime}}{\bar{\rho}} f_{r}^{o} .
\end{aligned}
$$

Eliminating $n^{i} u_{r r}^{i}$ from (4.43), we get

$$
\begin{aligned}
& \frac{2 \hat{\mu}+\hat{\mu}^{\prime}}{\bar{\rho}}\left(\frac{d \rho}{d t}\right)_{r}+p_{1} \rho_{r}=-n^{i} u_{t}^{i}+\frac{1}{J^{2}}\left\{\left(A^{2}+B^{2}\right) n^{i} u_{\psi \psi}^{i}+2(A C+B D) n^{i} u_{\psi \phi}^{i}\right. \\
& \left.\quad+\left(C^{2}+D^{2}\right) n^{i} u_{\phi \phi}^{i}-J\left(A e_{1}^{i}+B e_{2}^{i}\right) u_{\psi r}^{i}-J\left(C e_{1}^{i}+D e_{2}^{i}\right) u_{r \phi}^{i}\right\} \\
& \quad+\text { first order terms of } u \text { and } \theta+n^{i} f^{i}+\frac{\hat{\mu}+\hat{\mu}^{\prime}}{\bar{\rho}} f_{r}^{0} .
\end{aligned}
$$

If we apply $\partial^{k} \partial_{r}^{l}(k+l=0,1,2)$ to (4.44), multiply it by $\chi_{j}^{2} \partial^{k} \partial_{r}^{l}(d \rho / d t)_{r}$ and $\chi_{j}^{2} \partial^{k} \partial_{r}^{l} \rho_{r}$, and integrate them respectively in the similar way as in Lemmas 4.6 and 4.9, we can have

Lemma 4.11. For $k+l=0,1,2$, it holds that 
where

$$
\begin{aligned}
& \left\|\chi_{j} \partial^{k} \partial_{r}^{l+1} \rho(t)\right\|^{2}+\int_{0}^{t}\left\|\chi_{j} \partial^{k} \partial_{r}^{l+1} \rho(s)\right\|^{2}+\left\|\chi_{j} \partial^{k} \partial_{r}^{l+1}\left(\frac{d \rho}{d t}\right)(s)\right\|^{2} d s \\
& \leqq C\left\{\|D \rho(0)\|_{k+l}^{2}+\int_{0}^{t}\left\|\chi_{j} \partial^{k+1} \partial_{r}^{l} D u\right\|^{2}+\left\|\chi_{j} \partial^{k} \partial_{r}^{l} u_{t}\right\|^{2}\right. \\
& \quad+\|\left(D(u, \theta)\left\|_{k+l}^{2}+\right\| f^{0}\left\|_{k+l+1}^{2}+\right\| f \|_{k+l}^{2}+\left|B_{j, k, l}\right| d s\right\},
\end{aligned}
$$

$$
B_{j, k, l}=\int_{\Omega} \chi_{j}^{2}\left(\partial^{k} \partial_{r}^{l+1}\left(\frac{d \rho}{d t}\right)-\partial^{k} \partial_{r}^{l+1} \rho_{t}\right) \partial^{k} \partial_{r}^{l+1} \rho d x,
$$

and here the summation is not taken for $k$ and $l$.

Last we have to get a lemma corresponding to Lemma 4.7. Evidently the statement of Lemma 4.7, $k+l=0$ holds also for the case of exterior domains, if we add the term $\|D u\|$ on the right hand side of the inequality, (cf. Lemma 4.3). Next operating $\chi_{j} \partial^{k}, k=1,2$ to Stokes equation (4.6) with (4.17) and $\left.u\right|_{\partial \Omega}=0$, we have

$$
\begin{aligned}
& \bar{\rho}\left(\chi_{j} \partial^{k} u^{i}\right)_{x_{i}}=\chi_{j} \partial^{k} f^{0}-\chi_{j} \partial^{k}\left(\frac{d \rho}{d t}\right)+\bar{\rho} \chi_{j, x_{i}} \partial^{k} u^{i}, \\
& -\hat{\mu}\left(\chi_{j} \partial^{k} u^{i}\right)_{x_{l} x_{l}}+p_{1}\left(\chi_{j} \partial^{k} \rho\right)_{x_{t}}=-\chi_{j} \partial^{k} u_{t}^{i}-p_{2} \chi_{j} \partial^{k} \theta_{x_{i}}+p_{1} \chi_{j, x_{i}} \partial^{k} \rho-\hat{\mu} \chi_{j, x_{l} x_{l}} \partial^{k} u^{i} \\
& -\hat{\mu} \chi_{j, x_{l}} \partial^{k} u_{x_{l}}^{i}+\chi_{j} \partial^{k} f^{i}-\frac{\hat{\mu}+\hat{\mu}^{\prime}}{\bar{\rho}} \chi_{j} \partial^{k}\left(\frac{d \rho}{d t}\right)+\frac{\hat{\mu}+\hat{\mu}^{\prime}}{\bar{\rho}} \chi_{j} \partial^{k} f_{x_{i}}^{0}, \\
& \left.\quad \chi_{j} \partial^{k} u\right|_{\partial \Omega}=0 .
\end{aligned}
$$

Thus we can apply Lemma 4.3 to (4.45) and consequently we have

Lemma 4.12. For $l=0,1,2$, we have

$$
\left\|D^{2+l} u\right\|+\left\|D^{1+l} \rho\right\| \leqq C\left\{\left\|u_{t}\right\|_{l}+\left\|\frac{d \rho}{d t}\right\|_{1+l}+\left\|f^{0}\right\|_{1+l}+\|f\|_{l}+\|D(u, \theta)\|\right\},
$$

and for $k=1,2, l+k=1,2$, we have

$$
\begin{aligned}
& \left\|\chi_{j} D^{2+l} \partial^{k} u\right\|+\left\|\chi_{j} D^{1+l} \partial^{k} \rho\right\| \\
& \leqq C\left\{\left\|u_{t}\right\|_{k+l}+\left\|\chi_{j} \partial^{k} \frac{d \rho}{d t}\right\|_{1+l}+\left\|f^{0}\right\|_{1+k+l}+\|f\|_{k+l}+\|D \rho\|_{k+l-1}\right. \\
& \left.\quad+\|D(u, \theta)\|_{k+l}\right\} .
\end{aligned}
$$

Combining the above Lemmas $4.1-4.4$ and $4.9-4.12$ as in (i)-(xv) for the half space, one can obtain the same inequality (4.35) and the same Lemma 4.8 modulo the replacement of

$$
\sum_{k=0}^{4}\left|A_{k}\right|+\sum_{k+l=0}^{2}\left|B_{k, l}\right| \text { by }\left|A_{0}\right|+\left|A_{1}\right|+\sum_{k=2}^{4}\left|A_{k}^{\prime}\right|+\sum_{j=1}^{N}\left\{\sum_{k=2}^{4}\left|A_{j, k}\right|+\sum_{k+l=0}^{2}\left|B_{j, k, l}\right|\right\} .
$$

Thus the proof of Theorem 1.1 is completed. 


\section{References}

1. Agmon, S., Douglis, A., Nirenberg, L.: Estimates near the boundary for solutions of elliptic partial differential equations satisfying general boundary conditions II. Commun. Pure Appl. Math. 17, 3592 (1964)

2. Cattabriga, L.: Su un problema al contorno relativo al sistema di equazioni di Stokes. Rend. Mat. Sem. Univ. Padova 31, 308-340 (1961)

3. Finn, R.: On the exterior stationary problem for the Navier-Stokes equations, and associated perturbation problems. Arch. Rat. Mech. Anal. 19, 363-406 (1965)

4. Heywood, J. G.: A uniqueness theorem for non-stationary Navier-Stokes flow past an obstacle. Ann. Scuola Norm. Sup. Pisa IV 6, 427-445 (1979)

5. Ladyzhenskaya, O. A.: The mathematical theory of viscous incompressible flow. New York: Gordon and Breach 1969

6. Matsumura, A.: An energy method for the equations of motion of compressible viscous and heatconductive fluids, Univ. of Wisconsın-Madison, MRC Technical Summary Report 2194, 1981

7. Matsumura, A., Nishida, T.: The initial value problem for the equations of motion of compressible viscous and heat-conductive fluids. Proc. Jpn. Acad. Ser. A, 55, 337-342 (1979)

8. Matsumura, A., Nishida, T.: Initial boundary value problems for the equations of motion of general fluids. Computing Methods in Applied Sciences and Engineering, V, ed. by Glowinski, R., Lions, F. L. North-Holland Publ. Comp. Amsterdam, 1982, 389-406. In: Proc. of 5th Internat. Symp. on Computing Methods in Appl. Sci. and Engin, Dec. 1982, INRIA, Versalities, France

9. Solonnikov, V. A.: A priori estimates for certain boundary value problems. Sov. Math. Dokl. 2, 723 (1961)

10. Tani, A.: On the first initial-boundary value problem of compressible viscous fluid motion. Publ. RIMS. Kyoto Univ. 13, 193-253 (1977)

Communicated by J. Glimm

Received October 15, 1982; in revised form January 19, 1983 